\title{
Régulation métacognitive du contrôle de la compréhension orale en FLE
}

\author{
María José Valiente JiméneZ \\ Departamento de Filología Moderna \\ Universidad de Alcalá
}

Reçu: 10 Juin 2007 / Accepté: 10 Juillet 2007

ISSN: $1697-7467$

\begin{abstract}
RESUME: L'article souligne l'importance du contrôle de la compréhension orale d'une langue étrangère dans un apprentissage en présentiel et en non-présentiel. Au moyen d'une intervention métacognitive, on analyse l'évolution de la prise de conscience de cette stratégie et l'on décrit quelques procédés des étudiants universitaires espagnols, débutants et faux débutants de langue française, grâce à des verbalisations rétrospectives. Les résultats obtenus montrent des modifications qualitatives de la conscience et des stratégies impliquées dans le contrôle de la compréhension. On valide statistiquement cette modification dans le groupe complet, et on signale des différences en fonction du niveau de connaissance de la langue.

Mots-clés: Stratégies d'apprentissage, métacognition, compréhension orale, français langue étrangère.
\end{abstract}

\begin{abstract}
In this paper the importance of monitoring the process of listening, both in presential and non-presential learning of a foreign language, is underlined. Evolution of awareness in the use of this strategy has been analyzed through a metacognitive approach. Some procedures used by two groups of Spanish university students with different listening abilities in French, have been identified through their retrospective verbalisations.

The results obtained show that qualitative changes occur both in awareness and in the strategies involved in monitoring of listening. The statistical significance of these changes has been validated in both groups and the differences due to their level of knowledge has been identified.
\end{abstract}

Key words: Learning strategies, metacognition, oral comprehension, French as a foreign language

\section{INTRODUCTION}

Le fait de vérifier, valider ou infirmer des hypothèses de compréhension orale pendant la réalisation d'une tâche en situation formelle d'apprentissage est l'une des stratégies métacognitives les plus difficiles à enseigner-apprendre. En effet, le contrôle de la compréhension orale (monitoring) suppose, d'une part, l'évaluation continue de ce qu'on comprend pour valider la cohérence des prédictions avant et pendant l'écoute ; ce contrôle implique, d'autre 
part, plusieurs opérations telles que la transformation, l'élaboration, l'expansion et l'entrant de l'information comme le rappelle Markman (1985 : 276): "on some difficulty discovered in integrating or relating one part of a passage to another. This requires inferential or constructive processing - the transforming, elaborating, expanding of incoming information".

Dans le processus interactif qui se déclenche entre l'auditeur et le texte pendant les interprétations successives, l'auditeur met en rapport le message avec ses connaissances du monde et du contexte ; par exemple, face à un document oral avec le titre Au restaurant comme structurant, l'étudiant possède une expérience vitale ainsi qu'une expérience académique (lexique prévisible, actes de parole, actions, interlocuteurs, etc.). Egalement, il active des connaissances déclaratives (des savoirs tels que les systèmes phonologique, syntaxique, etc.); des connaissances procédurales (des savoir-faire liés «au comment de l'action, aux étapes pour réaliser une action, à la procédure permettant la réalisation d'une action " (Tardif, 1992 : 50) dans un contexte de tâches réelles et des connaissances conditionnelles ou stratégiques (le choix entre les différentes stratégies cognitives, métacognitives, etc). Il est bien connu que le mécanisme d'apprentissage ne s'effectue que par l'interaction entre les connaissances déclaratives, procédurales (Anderson, 1996) et stratégiques (Tardif, 1992). Mais comment aider les apprenants à gérer ces interactions dans l'écoute d'un document oral à visée communicative? Comment les encourager à assurer le bon fonctionnement d'un apprentissage tellement complexe? Quels sont les mécanismes qui pourraient favoriser le contrôle personnel de la compréhension?

Les connaissances que l'auditeur peut potentiellement mettre en jeu ont besoin d'une régulation qui favorise le contrôle des processus d'apprentissage de l'écoute. Au moyen des activités de type métacognitif, la réalisation de la tâche est améliorée par l'emploi des stratégies et par une plus grande conscience des difficultés de compréhension orale (Vandergrift, 1997; Goh, 1999, 2000). Ces activités comportent la planification, le contrôle (monitoring) et l'évaluation de la tâche de compréhension orale. Mais comment introduire une démarche de réflexion sur ces composantes fondamentales du processus d'apprentissage dans les cours de Langue Française à l'Université ?

La présente contribution vise un premier volet, lié à la prise de conscience de la stratégie du contrôle de la compréhension orale chez mes étudiants et à sa modification, et un deuxième volet qui porte sur l'analyse des réflexions des étudiants afin de s'approcher de la description qualitative des mécanismes mis en œuvre, pendant l'emploi de cette stratégie, dans l'écoute des documents oraux en FLE.

Le travail qu'on présente par la suite fait partie d'une étude plus vaste sur la prise de conscience de l'emploi de l'ensemble des stratégies métacognitives (planification, évaluation et contrôle de la compréhension orale); et sur la pratique de ces stratégies avec des étudiants universitaires espagnols au moyen d'une intervention métacognitive (Valiente Jiménez, 2004b). Nous abordons ici le contrôle de la compréhension orale de 22 étudiants universitaires, débutants et faux débutants de langue française (FLE) pendant l'écoute de documents oraux, à partir d'une analyse de leurs réflexions concernant leurs compréhensions personnelles. Par conséquent, cette intervention métacognitive s'inscrit dans la perspective des travaux qui privilégient la prise de conscience des processus d'écoute (Goh, 1998, 1999, 2000 ; Vandergrift, 1997, 1999, 2002, 2003) dans sa dimension métastratégique (Mendelsohn, 1994), ce qui suppose à mon avis, le développement des stratégies d'apprentissage liées à ce que Gombert (1990) appelle des habilités métalinguistiques, dans la compréhension orale. 


\section{LES PARTICIPANTS}

Les étudiants qui participent dans cette étude, en première année de Philologie Anglaise et inscrits dans les deux matières consécutives de Langue Française, constituent un groupe très hétérogène, composé par des étudiants espagnols, débutants et faux débutants, en situation formelle d'apprentissage. L'organisation officielle des cours en présentiel (75\%) et en nonprésentiel $(25 \%)$ m'a semblé particulièrement adéquate pour l'introduction d'un programme métacognitif afin d'aider les étudiants en compréhension orale et de les rendre plus autonomes vis-à-vis de cette compétence. Par conséquent, débutants et faux débutants sont soumis aux mêmes objectifs, contenus et activités du programme officiel de langue et du programme métacognitif proposé. La durée de cette intervention étant de trente semaines, quinze semaines par matière, les étudiants suivent le programme officiel qui contient des activités de compréhension orale, entre autres, pendant trois heures par semaine, en présentiel, et pendant une heure de plus, en non-présentiel, qui est consacrée au programme métacognitif et que l'on organise de différentes manières : en tutorial, groupe entier, petits groupes, travail libre, etc.

\section{Démarche PédagogiQue}

L'intervention métacognitive comporte la mise en oeuvre d'une démarche pédagogique tenant compte des trois variables de la métacognition : la connaissance de la personne, la connaissance de la tâche et la connaissance des stratégies (Wenden, 1998). Les étudiants réfléchissent à plusieurs questions : leurs représentations ou croyances concernant l'apprentissage de la langue étrangère et l'accès à la compréhension orale, leurs styles cognitifs, leurs aptitudes, les objectifs communicatifs visés, le type d'écoute à réaliser, les stratégies employées etc. et ils méditent sur l'influence de ces facteurs dans l'apprentissage de la langue (Rubin, 1994 ; Cornaire, 1998).

Le programme métacognitif est composé d'un programme général et d'un autre plus spécifique concernant les stratégies d'écoute. Le programme général implique la réalisation de différents types d'activités : remplir des questionnaires (basés sur Wenden (1987), Nunan, (1989) et Cyr (1996)) qui les aide à l'analyse de leurs trajectoires personnelles et à l'autodiagnose de leurs styles d'apprentissage individuels ; faire des représentations graphiques du style d'apprentissage personnel et de celui d'un autre camarade du cours; réaliser un test d'aptitude linguistique, de raisonnement verbal (Bennett, Seashore, Wesman, 1999); écouter des documents oraux dans une autre langue étrangère (Mendelsohn, 1994) et analyser les différents matériels utilisés par les étudiants, à partir des fiches qu'ils doivent remplir (Gremmo, 1994¹), ainsi que les objectifs communicatifs et le type d'écoute susceptible de pouvoir être réalisée dans chaque texte. À la fin de chaque activité, on fait une mise en commun des résultats obtenus dans le groupe.

${ }^{1}$ Fiche de "Documents oraux" réalisée par Gremmo pour le Centre de Recherches de l'Université de Nancy II, cité par Holec, H. (1994). 'L'apprentissage autodirigé: Une autre offre de formation. Strasbourg: Conseil de l'Europe. 
Le programme métacognitif spécifique concerne directement les stratégies de compréhension orale et les étudiants suivent un programme qui introduit des activités de planification, contrôle et évaluation de la compréhension (basé sur O’Malley et Chamot, 1990 et Vandergrift, 1999). Pour l'écoute de documents oraux en classe, ils réalisent des activités d'organisation préalable (Advance organization) à partir d'une feuille de réponse (questions à choix multiple, dessins avec des parcours à faire, localisation de différents endroits sur un plan, etc.) ou du titre du document comme le seul structurant de l'information. Au moyen des questions qu'on leur pose sur le sujet (Topic), sur les éléments de la situation de communication (actes de parole et vocabulaire prévisible compris), sur l'ordre de l'information dans le document, sur le type d'écoute à réaliser, etc., les étudiants préparent également des stratégies d'attention dirigée et d'attention sélective (Directed attention et Selective attention), ils décident de faire attention à des aspects concrets de l'input ou à des détails spécifiques de la situation de communication et d'ignorer l'information non pertinente. Tout de suite après la phase de planification de l'écoute, très importante pour le bon développement de la stratégie du contrôle de la compréhension, les étudiants sont invités à la vérification des hypothèses formulées auparavant, à la confirmation ou l'invalidation des ces hypothèses à partir de la recherche de la cohérence (entre leur interprétation et le contexte général de l'input, entre les différentes parties du document, etc.). Les hypothèses pouvant être d'ordre très divers, les étudiants sont portés au développement des capacités métalinguistiques : métalexiques, métasyntaxiques, métaphonologiques, métasémantiques et métapragmatiques (Gombert, 1990) et à prendre des décisions sur la valeur de différentes parties de l'input pour atteindre leurs objectifs de compréhension (real-time assessement of input, Goh, 1998a). Ils reformulent des hypothèses selon le type de confirmation de l'information (information générale, spécifique) et leur degré de validation (totale, partielle) à l'aide de contrôles auditifs et inférentiels. Finalement, les étudiants évaluent les résultats de leurs écoutes ainsi que la mise en oeuvre des stratégies employées.

Dans la première étape du programme métacognitif, le programme général, la réflexion des étudiants sur l'emploi personnel des stratégies porte sur l'ensemble des activités de compréhension orale (18 activités), réalisées dans les cours en présentiel. Les étudiants écoutent le texte trois ou quatre fois et tout de suite après, ils notent dans leurs cahiers des réflexions qu'ils doivent développer chez eux. Dans la deuxième étape, le programme métacognitif spécifique, ils portent leur attention sur l'écoute de 8 documents travaillés de manière autonome (en non-présentiel) avec les consignes de réfléchir avant et après l'écoute, d'écrire ces réflexions immédiatement après la tâche de compréhension et d'écouter et d'arrêter l'écoute des documents à plusieurs reprises s'ils le trouvent nécessaire. Au cours de la première étape, pour aider les étudiants à faire des descriptions réelles, on leur fournit des protocoles de réflexion d'après les suggestions de Goh (1998. b), à savoir, des éléments de réflexion pas trop spécifiques et l'évitement d'états hypothétiques dans les questions posées. Dans la deuxième étape de réflexion, avec les documents à travailler, les étudiants reçoivent des recommandations dans lesquelles on systématise l'ensemble des procédés utilisés dans la démarche méthodologique mise en pratique pendant les cours en présentiel. 


\section{Méthodologie de Recherche}

Les étudiants réalisent un cahier de bord écrit en espagnol à chaque étape, ainsi qu'un rapport au cours d'une épreuve de compréhension orale à la fin de la première 'étape. Pour mener à bien cette étude, on a dépouillé les journaux de bord des étudiants pour recueillir les données des verbalisations, selon le modèle d'Ericsson et Simons (1993). Les caractéristiques de cette intervention exigeait l'emploi de verbalisations rétrospectives tant pour les stratégies de planification (les étudiants décrivent comment ils préparent l'activité avant/pendant l'écoute des enregistrements) que pour les stratégies du contrôle de la compréhension (les étudiants font attention à l'input, ils déploient leurs opérations pour le traitement de l'information et ils commentent leurs processus après l'écoute). Les catégories utilisées sont basées sur le classement de Vandergrift (1999) et les travaux de Goh (1998a, 1998b). L'appartenance des étudiants à un sous-groupe ou à l'autre (débutants ou faux débutants) a été déterminée par les résultats d'une épreuve de compréhension orale et par les réponses des étudiants fournies par un questionnaire.

L'analyse des données des journaux des étudiants a été réalisée, en premier lieu, pour chercher des régularités dans la mise en oeuvre de la stratégie de contrôle de la compréhension avant et après l'intervention et en deuxième lieu, pour examiner des prévisibles changements qualitatifs des régularités dus au programme métacognitif appliqué. L'objectif de décrire des mécanismes sous-jacents dans la stratégie de contrôle: les stratégies cognitives impliquées (transferts, inférences), le type d'activité métacognitive réalisée (phonologique, syntaxique, sémantique, pragmatique, textuelle) ainsi que le poids de différentes composantes (la compétence linguistique, les traitements haut vers le bas etc.), m'a amené à choisir une analyse de type qualitatif; mais étant donné que dès le début de l'application du programme, la conscience des étudiants de la stratégie en question était très déficitaire, il m'a semblé très intéressant d'étudier le degré de modification de cette prise de conscience dans le groupe.

Les verbalisations des deux cahiers de bord ont été analysées et catégorisées. Cette catégorisation des données qualitatives étant par nature un processus subjectif (Goh, 1998.a) et pas toujours aisé, on a établit des critères très stricts, à savoir, l'acceptation des verbalisations du contrôle de la compréhension devait comporter 1) la description de l'emploi de la stratégie avec l'exemple concret des documents écoutés et 2) l'adoption de la terminologie adéquate (vérification, validation, invalidation, monitoring, etc).

Le test McNemar permet d'établir la différence entre deux traitements, dans le cas qui nous concerne, la méthode traditionnelle et l'intervention métacognitive. L'étudiant est considéré comme son propre contrôle car il est soumis aux deux traitements successifs; pour cette raison, le test est particulièrement approprié pour la validation statistique des données dans un type de description "avant-après » une intervention méthodologique (Siegel, 1976).

L'application du test McNemar étant très facile, l'on utilise une grille à quatre entrées de fréquences qui représente le premier et le second ensemble de verbalisations des mêmes étudiants (voir Tableau $\mathrm{n}^{\circ} 1$ ) ; l'on emploi + et - pour symboliser de différentes réponses $(+$ l'étudiant emploie la stratégie ; - il ne l'emploie pas). Les cas qui montrent des modifications entre la première verbalisation et la seconde apparaissent dans les cases A et D. Un étudiant est classé dans la case A s'il a changé de + à -, il est classé dans la case D s'il y a eu de modifications de - à + . Étant donné que $\mathrm{A}$ et $\mathrm{D}$ représentent le nombre total d'étudiants qui ont modifié leurs réponses, $1 / 2(\mathrm{~A}+\mathrm{D})$ serait la fréquence en ce qui concerne $\mathrm{H}^{\circ}$ (Hypothèse nulle). 
Tableau $n^{o}$ 1. Grille à quatre entrées pour la signification des modifications. Test McNemar.

\begin{tabular}{c|c|c|c|}
\multicolumn{3}{c}{} & \multicolumn{3}{c}{ Après } \\
& & - & + \\
\hline \multirow{4}{*}{ Avant } & + & A & B \\
\cline { 2 - 4 } & - & C & D \\
\cline { 2 - 4 } & &
\end{tabular}

Ensuite, l'on applique $c^{2}$ pour montrer l'existence d'une différence significative entre le nombre observé de réponses et le nombre attendu, basé sur l'hypothèse de nullité. La signification de la valeur observée de $\mathrm{c}^{2}$ est déterminée par la grille de Fisher et Yates abrégée (Siegel, 1976) (voir Tableau $n^{\circ} 2$ ).

\section{LA DESCRIPTION DU CONTRÔLE DE LA COMPRÉHENSION : DÈS TRACES À LA CONSCIENCE MÉTASTRATÉGIQUE}

Le manque de conscience des procédés du contrôle de la compréhension s'avère évidente lors d'une étude initiale, faite à partir des manifestations du premier cahier de bord. Il se peut que cette conscience « déficitaire » masque l'emploi réel de cette stratégie (Valiente Jiménez, 2004a). Dans le rapport écrit par les étudiants avant et après l'épreuve de compréhension orale, mentionnée ci-dessus, on observe que même s'il existe des élèves qui organisent préalablement leurs écoutes au moyen d'une synthèse de la situation de communication, ils n'explicitent pas la validation ou l'invalidation de leurs hypothèses ni même la vérification de la compréhension pendant l'écoute or, ceci dit, on peut analyser quelques symptômes récurrents qui permettent de déterminer l'emploi de la stratégie dans cette première 'étude. Mais en fait, il est très difficile de décrire le contrôle de la compréhension orale sans un entraînement métacognitif de l'étudiant.

Dans les paragraphes suivants, on va analyser, en premier lieu, les manifestations des étudiants liées au contrôle de la compréhension avant l'intervention métacognitive. Ensuite, on étudiera les références à l'emploi de cette stratégie pendant l'intervention et finalement, dans le paragraphe suivant, on commentera les résultats obtenus par l'application du test McNemar à la modification de la prise de conscience de la stratégie du contrôle grâce à l'intervention métacognitive.

\subsection{Les doutes, les corrections et l'estimation de l'input}

L'expression du doute et les corrections de la compréhension pendant l'écoute constituent des manifestations récurrentes du contrôle de la compréhension dans une première étape de réflexion. Les étudiants montrent une grande confusion et beaucoup de doutes à cause de leurs difficultés dans la compréhension du texte. Une élève écrit: "he querido confirmar la última pregunta pero todavía sigo con la duda de si (la respuesta) está bien o no" ("j'ai voulu confirmer la dernière question mais je continue à douter encore si la réponse est bien ou non »). Une autre étudiante évoque des doutes concernant plusieurs parties du document. 
Le contrôle de la compréhension peut donc porter sur une information précise ou sur plusieurs parties du texte oral.

On peut observer que l'ensemble des étudiants ont des doutes. Ce sont les élèves débutants (50\%) qui font référence à des hésitations générales alors que les élèves faux débutants expriment des doutes plus spécifiques $(75 \%)$. Il est évident que lorsqu'ils manifestent des indécisions plus générales, la possibilité de réussir dans le contrôle de la compréhension est plus difficile, ce qui pourrait montrer le poids de la compétence linguistique dans la construction des signifiés susceptibles de satisfaire des objectifs communicatifs. On peut également confirmer que le traitement haut vers le bas a besoin d'un input minimal dans la langue cible. À cet effet, le cas d'une élève débutante qui traduit le mot clé du document: travail par viaje (voyage, confusion avec le mot anglais travel) est significatif (Valiente Jiménez, 2003); ce transfert erroné provoque toute une série d'inférences également erronées qui font que le contrôle de la compréhension soit nécessairement négatif. Le manque de bagage linguistique suffisant chez l'étudiante empêche la prise d'indices nécessaire pour surmonter l'incohérence entre les différentes interprétations qu'elle réalise du texte:

si es un viaje nuevo o lo ha hecho otra vez, ¿por qué le pregunta esos datos tan específicos?; si está en un aeropuerto, en una agencia o en un teléfono, ¿por qué le repite esos números al final? (si c'est un nouveau voyage ou il l'a déjà fait, pourquoi il lui demande ces données si spécifiques? s'il est dans un aéroport, dans une agence ou au téléphone, pourquoi il lui répète ces numéros à la fin ?) (il s'agit en réalité d'une d'interview radiophonique d'un demandeur d'emploi).

Les corrections de la compréhension effectuées par les étudiants pendant l'écoute des activités fournissent des éléments pour comprendre le contrôle de la compréhension. Les élèves invalident une information vide de sens, un contresens, suite à un contrôle auditif ou inférentiel. Parfois ces corrections montrent des validations partielles des informations très précises : "corregí el número porque luego oí soixante» ("j’ai corrigé le numéro parce que j'ai entendu soixante après"), alors, le contrôle auditif de la compréhension corrige une audition déficitaire et implique des activités de discrimination de phonèmes et d' hétérophonie, ce qui suppose l'emploi de traitements de compréhension linguistiques (bas vers le haut).

Un autre type de correction révèle une succession de stratégies inférentielles. Quelques étudiants comprennent tout d'abord que le demandeur d'emploi veut travailler à niveau international, mais cette information est ensuite corrigée par l'information apportée dans une autre partie du document : pour des français à l'étranger. Dans ce cas, le contrôle de la compréhension est réglé grâce à l'input de différentes parties du document, au moyen des inférences des informations données tout au long du texte oral : "la opción pour des français à l'étranger se corresponde mejor con el resto del texto ", "lo he deducido por la información posterior ", ("l'option pour des français à l'étranger correspond mieux au reste du texte", "je l'ai déduite de l'information postérieure"). Voilà un bon exemple de transformation, d'élaboration et d'expansion de l'information pour la recherche de la cohérence. Des auditeurs qui n'ont pas réussi à surmonter une surdité ou qui ont échoué dans le contrôle auditif du mot français emploient le contrôle de la compréhension combiné avec des stratégies de haut rang pour compenser une carence dans la phase perceptuelle.

On peut constater que les étudiants débutants corrigent très peu leur compréhension (38\%). Deux élèves emploient un contrôle de la compréhension de type auditif et ils réussissent 
l'épreuve. Au contraire, la seule étudiante qui implique des inférences (erronées) dans le contrôle, ne réussit pas l'examen. Les étudiants faux débutants corrigent beaucoup plus leurs compréhensions (65\%). Pour la plupart, les contrôles sont également auditifs et leurs résultats sont acceptables. Les élèves qui ont fait référence à des stratégies inferentielles ont obtenu les meilleurs résultats de l'épreuve de compréhension.

En ce qui concerne l'estimation de l'input en temps réel (real-time assessment of input) qui est considérée comme l'une des stratégies de contrôle de la compréhension " because it involves noticing problems during listening and deciding what to do about them ", (Goh, 1998.b : 137), les étudiants évoquent un emploi total (100\%). La mise en oeuvre de cette stratégie pendant l'écoute du document leur permet de décider la valeur de l'input de différentes parties du texte par rapport aux objectifs de compréhension proposés. Les étudiants mettent de côté quelques parties du document pour privilégier " la información que da el hombre", (" l'information que l'homme donne",) et isoler "dentro de ésta las palabras estratégicas" (" dans celle-ci les mots stratégiques").

\subsection{La confirmation des hypothèses, la cohérence interprétative et les doutes}

Dans le deuxième cahier de bord, la plupart des étudiants expriment leurs contrôles de la compréhension au moyen de la vérification des hypothèses ; ils vérifient la cohérence de leurs interprétations et ils continuent à signaler des doutes personnels. Ils décrivent leurs emplois d'après leurs metastrategics awareness (ils donnent des exemples d'emploi de la stratégie en utilisant le langage métastratégique).

Les apprenants manifestent fondamentalement un contrôle de la compréhension dont l'objectif est la confirmation des hypothèses qu'ils formulent avant l'écoute. Dans cette première confirmation de type général, les hypothèses sont validées ou infirmées de manière totale ou partielle. Une élève débutante écrit:

Presté atención para ver si mi hipótesis se confirmaba o no. Me acerqué en el sentido de que cliente y dependiente iban a hablar sobre un producto (que no he entendido) y también cuando dije que el cliente compraría quizás varias cosas (papel de carta, cuaderno de notas) ; en lo que me equivoqué fue en lo referente a los precios, que no han sido nombrados... ( $j$ 'ai fait attention pour voir si mon hypothèse se confirmait ou non. Je me suis approchée dans le sens que le client et le vendeur allaient parler sur un produit (que je n'ai pas compris) et aussi lorsque j'ai dit que le client achèterait plusieurs choses (papier à lettres, un bloc-notes) ; je me suis trompée en ce qui concerne les prix qui n’ont pas été nommés)

Une autre étudiante affirme que son hypothèse est à moitié validée :

en efecto, se trataba de una guía turística que va explicando los diferentes edificios y monumentos a ambos lados del Sena, pero no he acertado en el recorrido » ( en effet, il s'agissait d'une guide touristique qui est en train d'expliquer des bâtiments et des monuments divers des deux côtés de la Seine, mais je n'ai pas deviné le parcours) 
Dans l'exemple suivant, l'étudiant invalide une partie de son hypothèse de travail sur le document oral :

Pensé que era un monólogo en el que alguien indicaba algo, al norte o al sur, a la derecha o a la izquierda, pero mi hipótesis no era correcta, en realidad el documento es un diálogo entre dos jóvenes. La chica le pregunta al chico por un lugar dentro de la facultad y él le va indicando dónde están las diferentes secciones ( j'ai pensé que c'était un monologue dans lequel quelqu'un indiquait quelque chose, au nord ou au sud, à droite ou à gauche, mais mon hypothèse n'était pas correcte, en réalité le document est un dialogue entre deux jeunes. La fille demande au garçon sur un lieu dans la faculté et lui, il lui indique où se trouvent les différentes sections)

Les vérifications des hypothèses faites préalablement par les étudiants, sont rapportées dans le cahier de bord par une bonne partie du groupe faux débutant $(75 \%)$ et à une moindre échelle, par le groupe débutant (60\%). Les différences entre les deux groupes sont également manifestes lorsqu'on analyse la fréquence de cet emploi dans l'ensemble des documents travaillés par les élèves, que ce soit des documents sans ou avec structurants (images, questions à choix multiple, etc.).

La prise de conscience de cette stratégie étant importante, on observe des différences considérables entre les deux groupes. Le décalage entre cet emploi et celui qui a été manifesté par rapport aux organisateurs et aux formulations d'hypothèses (débutants : 100\% ; faux débutants : 93\% ; groupe entier : 95\%) me semble également remarquable. Cela montre la difficulté de l'ensemble du groupe pour vérifier des hypothèses formulées auparavant, et notamment des débutants, ce qui pourrait confirmer, encore une fois, le poids de la compétence linguistique dans l'emploi des stratégies de compréhension orale. Comme Hédiart (1997:148) le remarque :

... tant que l'étudiant n'a pas atteint un certain niveau de connaissance de la langue, la prise d'indices qui lui permet de confirmer ou infirmer ses attentes formelles ou sémantiques de manière quasi-simultanée se fait de façon déséquilibrée: ses stratégies d'accès au sens sont limitées par une difficulté à vérifier ses hypothèses en recourrant aux signifiants du message.

De même, on observe 1) que la fréquence de l'emploi du contrôle de la compréhension est plus élevée dans le groupe des faux débutants (45\%) que dans le groupe débutant (35\%); 2) que la vérification des hypothèses augmente dans les documents sans structurants et 3) que l'écart entre les deux groupes se creuse dans ces documents. Par conséquent, on pourrait conclure que face à une plus grande difficulté (absence de structurants) la totalité des étudiants développent davantage cette stratégie et les difficultés des débutants s'intensifient vis-à-vis les faux débutants.

La vérification des hypothèses est directement liée à la recherche de la cohérence contextuelle, cependant, un petit nombre d'étudiants mentionnent la cohérence de ce qu'ils comprennent (27\%). D'après leurs explications, on peut observer que la plupart des étudiants visent la cohérence pour contraster le sens de ce qu'ils interprètent et leurs prior knowledge (Goh 1998a). Très peu d'élèves signalent la cohérence entre leurs interprétations de différentes parties du document oral ou des inférences du vocabulaire associé au sujet qu'on travaille. 
À mon avis, le fait d'une plus grande recherche de cohérence entre leurs interprétations et leur prior knowledge qu'avec les différentes informations du texte, dénote une étape encore très floue dans la radiographie des documents. Le nombre de références à l'incohérence de la compréhension étant aussi réduit (10\%), et elles se manifestent dans le même sens.

Les étudiants continuent à exprimer leurs doutes et on peut constater la même tendance signalée dans l'analyse du premier cahier de bord, avant l'intervention métacognitive (68\% des élèves y font référence, $75 \%$ débutants et $64 \%$ faux débutants). Les hésitations générales sont associées au groupe débutant (63\%) alors que les indécisions plus spécifiques appartiennent au groupe faux débutant (50\%). En ce qui concerne le type de document, les doutes, aussi bien généraux que spécifiques, sont davantage mentionnés lorsque les étudiants travaillent les documents sans structurants.

\section{LA PRISE DE CONSCIENCE DU CONTRÔLE DE LA COMPRÉHENSION}

Tout au long de cette description, on a pu constater une évidente modification de la prise de conscience de la stratégie du contrôle de la compréhension des étudiants. Cette transformation a été observée au niveau de la qualité des réflexions (apparition et développement de la vérification des hypothèses et de la cohérence interprétative) et au niveau du nombre d'étudiants qui font référence à ladite stratégie pendant l'intervention métacognitive. Les résultats de l'application du test McNemar m'ont permis valider statistiquement ce dernier type de modification. La prise de conscience du contrôle de la compréhension devient significative dans l'ensemble du groupe avec le degré maximum (0.0005) ; dans le groupe faux débutant, elle obtient un haut degré de signification (0.005) et dans le groupe débutant, le degré de signification diminue (0.05), comme l'on peut apprécier dans la Tableau $n^{\circ} 2$.

L'emploi de cette stratégie s'est étendu à un nombre important d'étudiants tant dans le groupe débutant que dans le groupe faux débutant. Il diffère néanmoins d'un groupe à l'autre. La différence pourrait s'expliquer par le fait que le contrôle de la compréhension soit une stratégie supérieure qui dirige d'autres stratégies métacognitives (prédiction, attention sélective) et des stratégies cognitives comme l'élaboration et l'inférence (Vandergrift, 1998) ; également, le moindre échantillonnage du groupe débutant peut creuser le fossé entre les deux sousgroupes (dans le test McNemar le degré de signification est directement lié au nombre d'individus). En tout cas, alors que cette stratégie n'était quasiment pas évoquée dans les premiers cahiers, les élèves l'ont incorporée à leurs réflexions à tel point qu'ils l'ont rendue statistiquement significative.

Tableau $n^{o} 2$. Application du test McNemar pour la validation des données par rapport à la prise de conscience du contrôle de la compréhension.(n $20: 8$ débutants et 14 faux débutants)

\begin{tabular}{|c|c|c|c|c|c|}
\hline & A & D & $\square^{2}$ & $\square$ & S.E. \\
\hline Groupe Entier & 0 & 14 & 12.07 & 0.0005 & S**** \\
\hline Débutants & 0 & 5 & 3.2 & 0.05 & S** \\
\hline Faux Débutants & 0 & 9 & 7.1 & 0.005 & S*** \\
\hline
\end{tabular}

(A: étudiants qui ont perdu la stratégie après l'intervention; D: étudiants qui ont acquis la stratégie; $\chi^{2}$ : valeur 1 de liberté; a: probabilité; S.E.: signification statistique) 


\section{Conclusion}

À l'heure actuelle, on ne conteste plus que la prise de conscience de l'emploi des stratégies personnelles soit l'une des parties essentielles de la métacognition (Wenden, 1987, 1991, 1998), bien que la description de la prise de conscience de cet emploi ne soit pas facile à faire, notamment en compréhension orale, par la difficulté inhérente à cette activité : "le caractère fuyant du document oral " (Hédiart, 1997 :150). Par ailleurs, les étudiants ne sont pas habitués à réfléchir sur leurs stratégies, ce qui représente une limitation de plus. Au début de cette intervention métacognitive, les élèves participants ont manifesté un manque d'emploi des mécanismes de contrôle de la compréhension orale qui pourrait être conditionné par un manque de conscience. Dans ce sens, grâce à cette intervention, on a pu déterminer une modification significative de la prise de conscience, validée par le test McNemar, dans l'ensemble du groupe. On a également établi des différences par rapport au degré de signification de celle-ci, en fonction du niveau de connaissance de la langue (débutants ou faux débutants) (Tableau $\mathrm{n}^{\circ}$ 2).

Les analyses des exemples extraits des cahiers de bord de mes étudiants m'aident à considérer que, dans ce genre d'études, importe non seulement le nombre d'étudiants qui réfèrent l'emploi des procédés de contrôle de la compréhension, la qualité et les combinaisons des stratégies impliquées (Vandergrift, 1998) mais aussi le degré de connaissance métastratégique. À cet effet, l'intervention métacognitive favorise également des modifications qualitatives de la conscience de l'emploi de cette stratégie, au moyen de la vérification des hypothèses, de la recherche de la cohérence interprétative, etc. et de leur métalangage spécifique.

Markman (1985) signale la frustration des étudiants quand ils trouvent des nonsequiturs dans le matériel utilisé ou dans la prémisse incohérente d'un argument difficile à suivre. Il est évident que, dans ces circonstances, quelque chose est erronée dans le matériel ou dans l'interprétation qu'on en fait. L'évaluation continue implique la confirmation des prédictions faites au moment de la planification de la compréhension orale et lors des synthèses successives de la situation de communication. Le contrôle de la compréhension orale est donc lié aux stratégies de planification telles quelles l'attention dirigée (Directed Attention), l'activation de la connaissance du contexte (Activate Background Knowledge), l'attention sélective (Selectively Attend) et la prédiction (Predict). Cependant, on observe les difficultés des étudiants, et notamment des débutants, pour valider les hypothèses qu'ils ont formulées au cours de la planification des documents. Même si la formulation des ces hypothèses signifie que les élèves s'appuient moins sur la composante linguistique et qu'ils deviennent autonomes en jouant un rôle plus interactif avec le texte (Gremmo, 86-87), leur niveau de connaissance de la langue influence énormément la stratégie du contrôle de la compréhension.

Les explicitations des procédés utilisés par les étudiants me permettent de remarquer des différences qualitatives dans la mise en oeuvre de ces procédés et leur combinaison. On pourrait se demander si le contrôle auditif basé sur la discrimination erronée des phonèmes dans une information précise a le même poids qu'un contrôle de type inférentiel entre les informations fournies dans les différentes parties du texte. On remarque parfois qu'une surdité dans un mot entraîne l'incompréhension d'une information et pourtant celle-ci peut être saisie grâce au contrôle de la compréhension, combiné avec des stratégies inférentielles portant sur plusieurs parties du document. Mais quelquefois, un signifiant du message est non seulement mal segmenté et mal discriminé, mais il échappe de surcroît à l'opération d'interprétation et 
laisse un vide de sens ; l'élève se bloque dans cette phase perceptuelle et ne réussit pas à employer des stratégies qu'il pourrait bien avoir à sa disposition. En tout cas, la nécessité d'insister sur les deux types d'approches (haut vers le bas et bas vers le haut) semble indispensable du point de vue pédagogique pour aborder la complexité de la compétence de compréhension orale.

Pour finir, bien que les limitations de cette étude (nombre de participants, inégalité des sous-groupes, impossibilité d'observer toutes les activités mentales au moyen des verbalisations, un seul support (audio), etc.) ne permettent pas la généralisation des résultats qui devront être confirmés par d'autres études plus larges, l'objectif d'améliorer la pratique quotidienne et autonome de mes élèves dans la compréhension orale a été largement atteint grâce à l'intervention métacognitive; en outre, elle me permet non seulement d'augmenter de manière significative la prise de conscience et le développement de la connaissance métastratégique chez mes étudiants, mais aussi de réfléchir sur quelques procédés impliqués dans le contrôle de la compréhension orale. À mon avis, la métacognition s'avère incontournable comme méthode pour pouvoir atteindre une description réelle des mécanismes de compréhension des étudiants en FLE.

\section{REFERENCES BIBLIOGRAPHIQUES}

Anderson, J.R. (1996). «ACT : A Simple Theory of Complex Cognition", American Psychologist, $51: 355-365$.

Bennett, G.K.; Seashore, H.G. et Wesman, A.G. (1999). DAT Test de Aptitudes Diferenciales. Madrid: TEA.

Cornaire, C. (1998). La Compréhension orale. Paris : Clé International.

Cyr, P. (1996). Le point sur les stratégies d'apprentissage d'une langue seconde. Québec : Les Éditions CEC.

Ericsson, K.A. et Simons, H.A. (1993). Prototocol Analysis: VerbalRreports As Data . Cambridge: MIT Press.

Goh, C. (1998.a). Strategic Processing and Metacognition in Second Language Listening. PhDthesis. Lancaster University.

Goh, C. (1998.b). "How ESL Learners with Different Listening Abilities Use Comprehension Strategies and Tactics". Language Teaching Research 2, 2: 124-147.

Goh, C. (1999). " How Much Do Learners Know about the Factors That Influence Their Listening Comprehension”. Hong Kong Journal of Applied Linguistics 4, 1: 17-40.

Goh, C. (2000). " Cognitive Perspective on Language Learner's Listening Comprehension Problems". System $28: 55-75$.

Gombert, J.E. (1990). Le développement métalinguistique. Paris: P.U.F

Gremmo, M.J. (1986-87) “ À propos d'écoute...écoute”. Mélanges pédagogiques 86/87 : 55-69.

Hédiart, M. (1997). "Représentation orale et écrite du signifiant.” In M. Maillard, et L. Dabène (eds.), Vers une métalangue sans frontières? Lidil 14 : 145-154.

Markman, E.M. (1985). “Comprehension Monitoring”. In S.F. Chipman, J.W. Segal et R. Glaser (eds.), Thinking and Learning Skills 2: 275-291.

Mendelsohn, D. (1994). Learning to Listen: A Strategy-based Approach the Second Language Learner. San Diego: Dominie Press. 
Nunan, D. (1989). Understanding Language classrooms - A Guide for Teacher Initiated Action. H. Hempstead : Prentice-Hall.

O’Malley, J.M. ; Chamot, A.U. (1990). Learning Strategies in Second Language Acquisition. Cambridge : Cambridge University Press.

Rubin, J. (1994). "A Review of Second Language Listening Comprehension Research", The Modern Language Journal, 78: 199- 221.

Siegel, S. (1976). Estadística no paramétrica aplicada a las ciencias de la conducta. México: Trillas.

Tardif, J. (1992). Pour un enseignement stratégique: l'apport de la psychologie cognitive. Montréal: Les Editions Logiques.

Valiente Jiménez, M. J. (2003). “Transferencias e inferencias en la comprensión oral del francés en estudiantes universitarios". In N. Duchêne et M.J. Chaves (eds.) Identités culturelles francophones: de l'écriture à l'image. Servicio de Publicaciones de la Universidad de Huelva: 133-141.

Valiente Jiménez, M.J. (2004a). "Uso de la estrategia metacognitiva de planificación en la comprensión oral de una lengua extranjera". In A.M. Suárez; F. Ramallo et X.P. Rodríguez Yánez (eds.) Bilingual Socialization and Bilingual Language Acquisition: Proceedings from the Second Internacional Symposium on Bilingualism. Servicio de Publicaciones de la Universidad de Vigo: 781-792.

Valiente Jiménez, M.J. (2004b). "La conciencia del uso de estrategias de comprensión oral en L2, a través de una intervención metacognitiva” . In M. Díez, R. Fernández et A. Halbach (eds) Debate en torno a las estrategias de aprendizaje/Debating Learning Strategies. Frankfurt an Main: Peter Lang, 111-124.

Vandergrift, L. (1997). "The Strategies of Second Language (French) Listeners: A Descriptive Study", Foreign Language Annals 30: 87-409.

Vandergrift, L. (1998). "La métacognition et la compréhension auditive en langue seconde", Canadian Journal of Applied Linguistics 1, 1-2 : 83-105.

Vandergrift, L. (1999). "Facilitating Second Language Listening Comprehension: Acquiring Successful Strategies", ELT Journal, 53: 168-176.

Vandergrift, L. (2002). "It Was Nice to See that Our Predictions Were Right", Canadian Modern Language Review/La revue canadienne des langues vivantes, 58, 4 : 555-75.

Vandergrift, L. (2003). "Pleins feux sur la classe, de l'anticipation à la réflexion: Comment guider les élèves dans le processus d'écoute en langue seconde", The Canadian Modern Language Review/La revue canadienne des langues vivantes, 59, 3. http://www.utpjournals.com/ jour.ihtml?lp=product/cmlr/593/vandergrift.html.

Wenden, A. (1998). "Metacognitive Knowledge and Language Learning", Applied Linguistics 19, 4: 515-537. 